Research Article

\title{
Water Saving Control of Turfgrass Irrigation Robot Using Genetic Simulated Annealing Algorithm
}

\author{
Bei Han \\ School of Information Engineering, Yulin University, Yulin 719000, China \\ Correspondence should be addressed to Bei Han; hanbei@yulinu.edu.cn
}

Received 3 June 2021; Accepted 8 July 2021; Published 16 July 2021

Academic Editor: Fazlullah Khan

Copyright (C 2021 Bei Han. This is an open access article distributed under the Creative Commons Attribution License, which permits unrestricted use, distribution, and reproduction in any medium, provided the original work is properly cited.

\begin{abstract}
Artificial intelligence through the robotic system offers a solution to the quest for an autonomous system with high cutting efficiency for lawn mowing. Because of the current trimming and maintenance operations on grasslands and gardens, it is essential to develop autonomous and efficient lawn pruning electromechanical equipment. This paper describes the design and construction of a high-performance automated grass trimming and irrigating robot. This device cuts and irrigates grass automatically with little human intervention. A genetic simulated annealing algorithm was employed to optimize motor parameters, specifically design a set of mowing mechanisms and mowing height adjustment system. The prototype was tested, which mainly includes the running status evaluation of the walking module, the mowing module, the cutter head lifting module, and the collision detection module. This robot can save water while watering the lawns, reduce labor costs, and improve mowing efficiency. We note that the proposed system can be implemented on a large scale under natural conditions in the future, which will be helpful in robotics applications and cutting grass on lawns and playing grounds.
\end{abstract}

\section{Introduction}

Lawn mowing is the process of trimming grasses to a specific height, usually for sanitation, decoration, and sport purposes [1-3]. At present, lawn trimming and irrigating on the market mainly rely on manual operation, which has the disadvantages of large labor, large environmental pollution, and high repetitive operation. Moreover, it is not easy to adapt to the needs of the fast-developing lawn industry [4]. Various grass-cutting and irrigating equipment have been developed over the years, such as spiral-rope cutter, reelblade grass cutter, and gas-powered mower. However, they pose some challenges relating to operational efficiency. A lawnmower is a tool like scissors and a machine that consists of unified powered and electrical systems with an effective controlling mechanism.

With the introduction of intelligence in lawn mowing and irrigating robots, various intelligent lawn mowing robots have emerged in an endless stream. However, most of them have similar functions [5]. They can all automate functions such as starting, charging, mowing, and watering the grass. According to the degree of automation, the development of robots can be roughly divided into three stages: the initial stage of development, the intermediate stage of semi-intelligence development, and the current stage of higher intelligence $[6,7]$. The Bosch Indigo mowing robot produced in Germany has many improvements compared with the traditional grass industry robot. It has improved the original working model of random mowing [8]. The robot uses sequential rows to work. The unique grass-cutting path planning dramatically improves mowing efficiency. The Indigo mowing robot also has the function of independent charging [9-11]. After the charging is completed, it can autonomously return to the original stopped area to continue cutting. Grass homework, the lawnmower robot developed by Ohio University in the United States, is highly intelligent $[12,13]$. The overall structure is made of alloy materials, and a DC motor drives the motion device. The working speed can reach $16 \mathrm{~km} / \mathrm{h}$. In addition, the robot uses a differential global positioning system in the path planning 
process, which ensures the accuracy of the movement during the navigation process and improves the movement efficiency of the robot.

Derander et al. [14] designed a smart lawn grass trimming robot which does not require any boundary cable for its operation to reduce the cutting cycle time and operational cost. The author in [15] developed a mechanical push lawn with the double cylinder for blade spinning to increase the system's capacity and operational efficiency. Moore [16] performed the measurement and analysis of a lawnmower in terms of its performance and noise level. Many other works have been reported on the design of autonomous lawnmowers and systems. It requires installing boundary wire around the perimeter of the lawn to keep the machine within the predetermined area and on a path [17-19]. Moreover, some existing mowers use a random path that requires more time to mow with low cutting efficiency and do not produce the typical even and clean mowing lines which characterize a well aesthetically manicured lawn. Furthermore, some existing lawnmowers operate semiautonomously, relying on human intervention for some degree of operation, thus, increasing the cutting cycle time, human drudgery, and labor cost.

From the perspective of adapting to market demand, this article aims to develop a prototype for high-performance autonomous grass trimming and irrigating robot. This robot can save water while irrigating the lawns, reduce labor costs, and improve mowing efficiency. This means that the garden machinery manufacturing industry will face more opportunities. Furthermore, the proposed grass trimming robot can adapt to domestic development needs, increase international market share, and provide good economic and social benefits.

The rest of the paper is structured as follows. Section 2 provides an overview of irrigating and grass trimming robots' general architecture. Then, in Section 3, the simulated annealing algorithm is discussed. Then, Section 4 describes different results, and finally, the conclusion is given in Section 5.

\section{Architecture of the Grass Trimming Robot}

The successful development of intelligent lawnmower robots dramatically saves human resources and energy and reduces environmental pollution to a certain extent [14-16]. Compared with traditional hand-operated lawnmowers, the intelligent lawn mower robot has low noise and low dust characteristics. As a result, it reduces noise pollution to the surrounding environment. It has less impact on people's health and the environment $[17,18]$. The grass trimming and irrigating robot motion control system is comprised of three parts: the driving wheel motor, the cutter head motor, and the elevator motor. The robot's walking mechanism relies on the rear wheels on both sides to drive and perform stop, straight and turn movements by controlling the motor speed on the left and right sides $[19,20]$. The driving wheel uses a brushless DC motor drive system, which is convenient for the control and speed adjustment of the mobile platform. The rotary motion of the mowing blade is called the mowing mechanism of the mowing robot, which is required to regulate the speed of the motor and control start and stop. The schematic diagram of lawn grass robot control is shown in Figure 1.

The path planning method of mobile robots is based on the robot's specific control architecture and different environmental characteristics. For mobile robots with different environments, different planning methods have their advantages and disadvantages [21]. At present, there is no general planning method that can adapt to any system in various environments. Therefore, it is necessary to choose several different planning methods to combine their respective advantages to obtain the best planning effect [22]. In the overall design of the autonomous grass trimming and irrigating robot, this section will propose the design of the autonomous grass trimming robot. It clarifies the designed autonomous robot's functions and proposes a prototype according to actual requirements [23].

\section{Water-Saving Control Based on Simulated Genetic Annealing}

The simulated annealing algorithm is a random optimization algorithm based on the Monte Carlo iterative solution strategy. It has been widely used in engineering applications. During a Monte Carlo simulation, values are sampled randomly from the input probability distributions. Each set of samples is called an iteration, and the resulting outcome is recorded. The idea comes from the similarity between the annealing process of solid matter in physics and general combinatorial optimization problems. The temperature at the beginning of the simulated annealing algorithm is very high $[24,25]$. As the temperature continues to drop, it randomly searches for the optimal global solution of the objective function in the solution interval. This can ensure that the optimal local solution probabilistically jumps out and finally reaches the optimal global solution [26]. Theoretically, the algorithm has probabilistic global optimization performance. It can accept inferior solutions to a limited extent to not fall into the local optimum. The rule of thumb requires the algorithm to search for the most significant possible solution space in a reasonable time, with only a sufficiently large time $[27,28]$. This may lead to too long execution time, making the simulated annealing algorithm infeasible. The theoretical analysis points out that the algorithm should be quasi-balanced at the beginning.

$$
\mu=\left(L^{H} L\right)^{-1} L^{H} d .
$$

When the control parameter decays slowly, the two adjacent values are smoothly distributed and close.

$$
\mu=L^{H}\left(L^{H} L\right)^{-1} d .
$$

Therefore, on the one hand, if the quasi-balance is reached, only a small amount of transformation is sufficient to restore the quasi-balance. Therefore, a shorter length of the Markov chain can be selected to reduce the execution time $[29,30]$. 


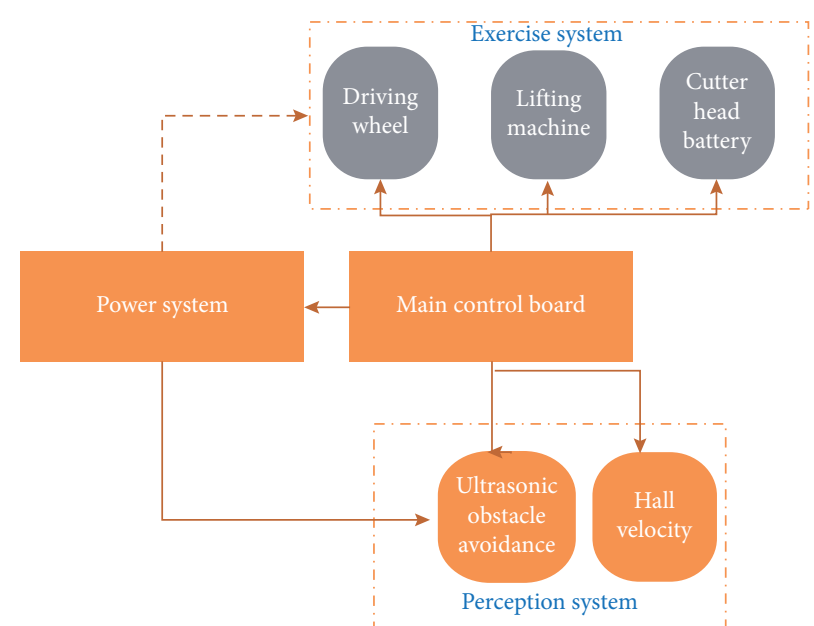

Figure 1: Schematic diagram of transmission line fault location.

On the other hand, a slight attenuation of the control parameter may also increase the number of iterations of the algorithm. It can be expected that the algorithm process will accept more transformations, search a more extensive range of solution spaces, and return a higher quality final solution.

$$
\mu(\tau, p)=\int_{-\infty}^{+\infty} d(t=\tau+p x, x) \mathrm{d} x .
$$

The main disadvantage of the simulated annealing algorithm is that it takes a long time to find a high-quality approximate optimal solution significantly when the problem size inevitably increases [31]. However, proper selection of the cooling schedule can effectively improve the performance of the algorithm.

$$
\begin{aligned}
d^{\prime}(t, x) & =\int_{-\infty}^{+\infty} \mu(\tau-p x, x) \mathrm{d} p \\
\mu(\tau, p) & =\sum_{i}^{N x} d\left(t=\tau+p x_{i}, x_{i}\right) \Delta x_{i} .
\end{aligned}
$$

We combine simulated annealing and genetic algorithm to provide a general framework for solving complex system optimization problems. It does not depend on the domain and type of the problem. The genetic algorithm is used to deal with the optimization problem. The feasible solution of the optimization problem corresponds to everyone in the group [32-34].

$$
a=\frac{\sum\left(y_{i} / \sigma_{i}^{2}\right) e^{b\left(x_{1 i}+x_{2 i}\right)}}{\sum\left(1 / \sigma_{i}^{2}\right) e^{2 b\left(x_{1 i}+x_{2 i}\right)}}
$$

The objective function of the optimization problem corresponds to the environment in which the group is located, and the function value corresponds to the fitness of the individual to the environment. The chromosomes are encoded in binary strings in the algorithm, and each encoded string is a candidate solution group. There are multiple chromosomes, that is, a group of candidate solutions [35].

$$
\sum \frac{y_{i}}{\sigma_{i}^{2}} e^{b\left(x_{1 i}+c x_{2 i}\right)}=\frac{\sum\left(1 / \sigma_{i}^{2}\right) e^{2 b\left(x_{1 i}+x_{2 i}\right)}}{\sum\left(\left(x_{1 i}+x_{2 i}\right) / \sigma_{i}^{2}\right) e^{2 b\left(x_{1 i}+x_{2 i}\right)}} \cdot \sum \frac{\left(x_{1 i}+x_{2 i}\right) y_{i}}{\sigma_{i}^{2}} e^{b\left(x_{1 i}+x_{2 i}\right)}
$$

Chromosomes are the main objects of evolution. Like biological evolution, crossover and mutation make the final solution global. For a practical application problem that requires optimization calculation, the genetic algorithm to solve the problem can generally be constructed according to the following steps:

$$
d^{\prime}(t, x)=\sum_{j}^{N_{p}} \mu\left(\tau=t-p_{j} x, p_{j}\right) \Delta p_{j}
$$

The simulated annealing algorithm has strong local searchability. It can jump out of the local minimum and tend to the global optimum $[36,37]$. In addition, it has good asymptotic convergence, simple description, flexible application, suitable for parallel calculation, and overcomes initial value dependence. The other advantages can effectively solve the problem with nondeterministic polynomial-time (NP) complexity [38, 39]. However, at the same time, the simulating annealing algorithm requires a higher 
initial temperature and a longer sampling step and a larger time.

$$
\begin{aligned}
\mu & =L^{H} d, \\
d^{\prime} & =L \mu .
\end{aligned}
$$

\section{Water-Saving Control Process Test and Analysis}

From the perspective of adapting to market demands, this article aims to develop high-performance autonomous grass trimming and irrigating robots [40, 41]. We performed experiments and analyzed the prototype, which mainly included the running status evaluation of the walking module, the mowing module, the cutter head lifting module, and the collision detection module [42].

4.1. Technical Performance Test. Technical performance testing is an indispensable part of the research process of autonomous irrigating and grass trimming robots. Tests are used to verify the feasibility of the overall scheme and test the reliability of each module of the prototype and the rationality of the mechanical structure design [43]. After the overall design plan was determined, the 3D modeling of the autonomous irrigating and grass trimming robot was carried out with solid works software. Figure 2 describes the simulation test data distribution. The distribution of data shows that the system has the best performance for water-saving and grass cutting. Figure 3 shows the test results of the vibration frequency of the grass-cutting and irrigating robot using four different methods [44].

The walking module is an integral part of the autonomous irrigating and grass trimming robot system. The highperformance walking system is an essential guarantee for the irrigating and grass trimming robot to complete the mowing work efficiently. The stability, accuracy, and flexibility of its actions will directly affect the robot's performance [45]. Figure 4 describes the water-saving control effect in three different grass lawns and environments when the frequency is $100 \mathrm{~Hz}$.

The robot provides the save performance even if the environment is changing and that the change of environment does not affect the efficiency of the proposed model. Figure 5 provides a comparison of the water-saving efficiency between the proposed optimized robotic machine and the ordinary machine. For 200 robot data samples, the water-saving rate of the proposed grass trimming and irrigating robot is $0.7 \%$, whereas that of the ordinary lawn robot is $0.6 \%$. Similarly, for 800 data samples, the proposed model has a water-saving rate of $0.8 \%$, which is greater than the ordinary machine having a water-saving rate of $0.5 \%$. This confirms that the proposed model has an improved water-saving effect compared with traditional irrigating and grass trimming robots.

In addition to static positioning, the lawnmower robot is based on differential positioning technology. Therefore, it needs to ensure that the robot changes the position dynamically during the working process, which requires the

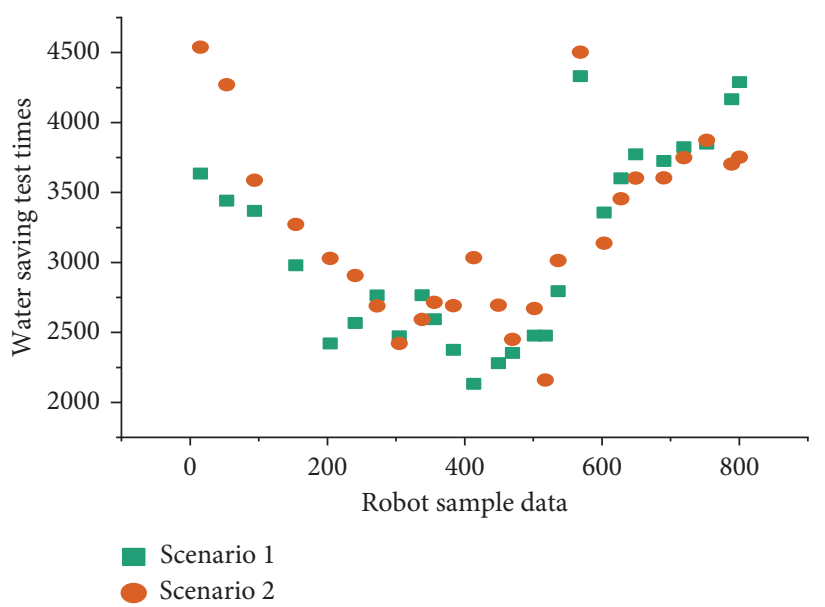

FIgURE 2: Simulation test data distribution irrigating and grass trimming robot system.

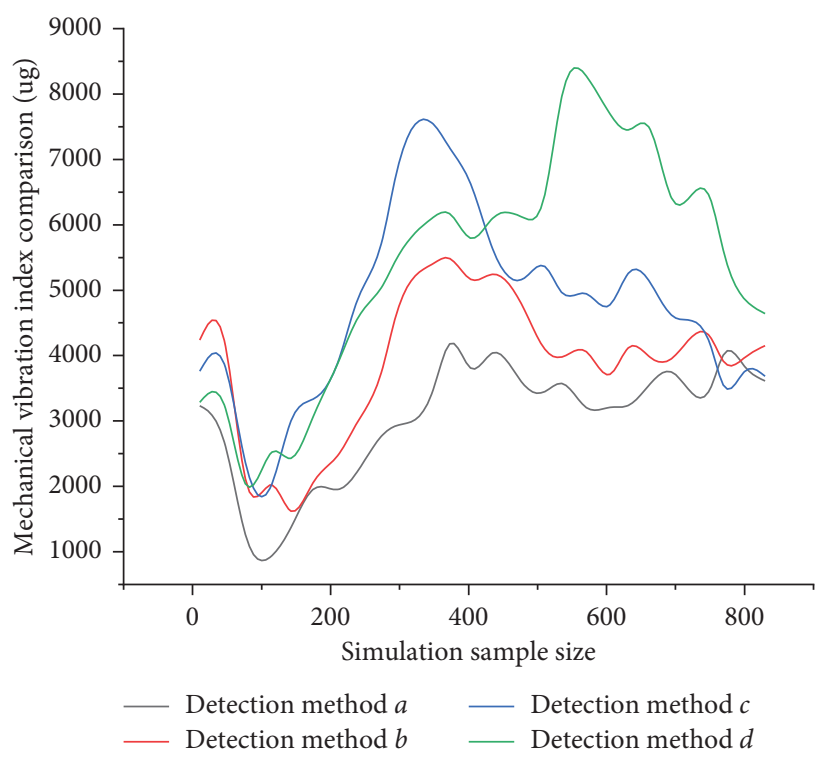

FIgURE 3: Vibration frequency test results of irrigating and trimming robot.

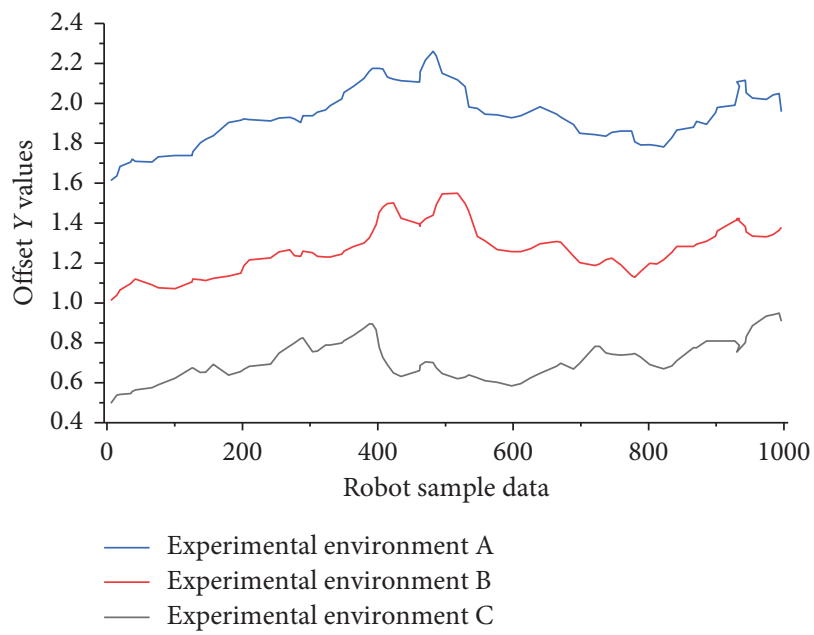

FIGURE 4: Water-saving control effect when the frequency is $100 \mathrm{~Hz}$. 


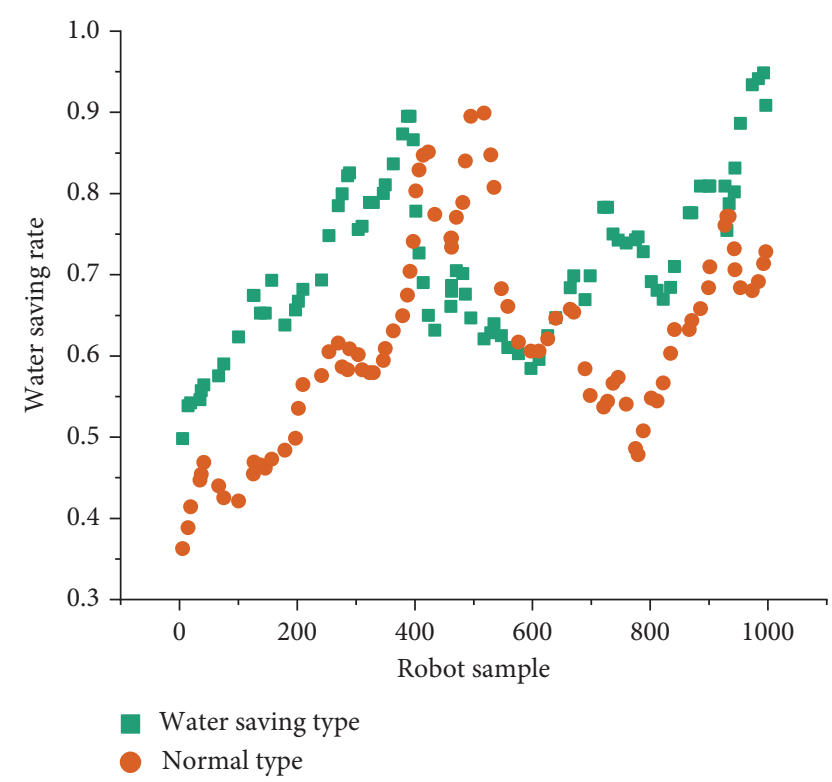

Figure 5: Comparison of water-saving efficiency between the proposed model and ordinary robotic machines.

system to achieve high dynamic accuracy. Compared with traditional hand-operated lawnmowers, the intelligent lawn mower robot has low noise and low dust characteristics. Therefore, it reduces noise pollution to the surrounding environment and has less impact on people and the environment. Although the mowing robot designed in this study is an outdoor mobile device, its evaluation in the real environment needs further verification.

\section{Conclusion}

This article comprehensively and profoundly analyzes the actual market demand of trimming and watering robots and carefully studies the current development status of automatic lawnmower robots. A compact and economically feasible overall plan for an autonomous grass trimming and irrigating robot is proposed after a comprehensive study of the plan of the autonomous robot based on the dynamics and kinematics analysis of the outdoor wheeled mobile robot. It is combined with the work requirements of the grass trimming and irrigating robot. The overall layout and car body of the robot are studied. This paper describes the design and construction of a high-performance automated grass trimming and irrigating robot. A genetic simulated annealing algorithm was used to enhance motor parameters, specifically design a set of mowing apparatus and mowing height adjustment system. The prototype was evaluated, including the running status evaluation of the walking module, the mowing module, the cutter head lifting module, and the collision detection module. The proposed robot can save water while watering the lawns, reduce labor costs, and improve mowing efficiency. The proposed system will be implemented on a large scale under real conditions in the future. It will be useful in robotics applications and cutting and irrigating grass on lawns and playing grounds.

\section{Data Availability}

The data used to support the findings of this study are available from the corresponding author upon request.

\section{Conflicts of Interest}

The author declares that there are no conflicts of interest.

\section{References}

[1] J. S. Powlen, C. A. Bigelow, A. J. Patton, Y. Jiang, and M. L. Fraser, "Minimal irrigation requirements of Kentucky bluegrass and tall fescue blends in the northern transition zone," Crop Science, vol. 2, 2020.

[2] M. Hong and D. J. Bremer, "Minimum water requirements of Japanese lawngrass (zoysia japonica steud) for survival during prolonged drought," Crop Science, vol. 3, 2020.

[3] Y. Takefuji, "Adopt the latest flood-resistant and wildfiresresistant irrigation technology," Science, vol. 9, 2018.

[4] S. Zhang, J. Zhang, T. Yue, and X. Jing, "Impacts of climate change on urban rainwater harvesting systems," Science of The Total Environment, vol. 665, pp. 262-274, 2019.

[5] G. Simone, L. Misuraca, U. Anceschi et al., "Urethra and ejaculation preserving robot-assisted simple prostatectomy: near-infrared fluorescence imaging-guided madigan technique," European Urology, vol. 75, no. 3, pp. 492-497, 2019.

[6] S. Raheem, Y. E. Ahmed, A. A. Hussein et al., "Variability and interpretation of communication taxonomy during robotassisted surgery: do we all speak the same language?" $B J U$ International, vol. 122, no. 1, pp. 99-105, 2018.

[7] M. Shahait, K. Patel, S. Na et al., "Stepwise description and outcomes of bladder neck sparing robot-assisted simple prostatectomy," Journal of Endourology, vol. 34, no. 5, pp. 588-593, 2020.

[8] S. Yang, X. Sun, J. Ding, Z. Jiang, X. Liu, and J. Xu, "Effect of biochar addition on $\mathrm{CO}_{2}$ exchange in paddy fields under water-saving irrigation in southeast China," Journal of Environmental Management, vol. 271, Article ID 111029, 2020.

[9] Y. Xu, F. Jiang, J. Du, and D. Gong, "A cross-domain collaborative filtering algorithm with expanding user and item features via the latent factor space of auxiliary domains," Pattern Recognition, vol. 94, pp. 96-109, 2019.

[10] M. T. Uddin and A. R. Dhar, "Assessing the impact of watersaving technologies on boro rice farming in Bangladesh: economic and environmental perspective," Irrigation Science, vol. 38, pp. 199-212, 2020.

[11] C. Gao, W. G. Gao, K. Song, H. Na, F. Tian, and S. Zhang, "Comprehensive evaluation on energy-water saving effects in iron and steel industry," Science of the Total Environment, vol. 670, pp. 346-360, 2019.

[12] Y. Xu, Y. Chu, F. Jiang, Y. Guo, and D. Gong, "SVMs classification based two-side cross domain collaborative filtering by inferring intrinsic user and item features," KnowledgeBased Systems, vol. 141, pp. 80-91, 2018.

[13] W. Petros, G. B. Tesfahunegn, M. Berihu, and J. Meinderts, "Effectiveness of water-saving techniques on growth 
performance of mango (mangifera indical) seedlings in mihitsab-azmati watershed, rama area, northern Ethiopia," Agricultural Water Management, vol. 243, 2021.

[14] J. M. Derander, P. Andersson, E. Wennerberg, A. Nitsche, E. Moen, and F. Labe, "Smart robot lawn mower," pp. 1-70, Department of Computer Science and Engineering, Chalmers University of Technology, University of Gothenburg, Gothenburg, Sweden, 2018, Bachelor thesis.

[15] C. S. Namoco, J. D. Achas, R. Alcantara, J. Anora, and C. C. Buna, "Development of a mechanical push lawn mower with double-cylinder spinning blades," International Journal of Engineering Research and Technology, vol. 2, no. 8, pp. 1861-1863, 2013.

[16] M. D. Moore, "Measurement and analysis of lawn mower performance and noise," pp. 1-98, Iowa State University, Ames, IA, USA, 1997, Masters thesis.

[17] X. Yu, D. Zhan, L. Liu, H. Lv, L. Xu, and J. Du, “A privacypreserving cross-domain healthcare wearables recommendation algorithm based on domain-dependent and domainindependent feature fusion," IEEE Journal of Biomedical and Health Informatics, vol. 6, p. 1, 2021.

[18] K. LaMott and S. Tosunoglu, "Development of a robotic lawnmower as an adaptable platform for swappable remote and autonomous control packages," in Proceedings of the 2010 Florida Conference on Recent Advances in Robotics, Jacksonville, Florida, May 2010.

[19] S. H. Bhutada and G. U. Shinde, "Design modification and performance comparison of lawn mower machine by mulch and flat type cutting blade," International Journal of Agricultural Sciences, vol. 9, no. 40, pp. 4638-4641, 2017.

[20] D. F. Hauser, R. Mailig, H. Stadtler, J. Reed, and K. Bernd, "Waterpipe tobacco smoke toxicity: the impact of waterpipe size," Tobacco Control, vol. 29, no. Suppl 2, Article ID 054960, 2019.

[21] Y. Xu, J. Yang, and Z. Xie, "Training SVMs on a bound vectors set based on Fisher projection," Frontiers of Computer Science, vol. 8, no. 5, pp. 793-806, 2014.

[22] L. G. Raeisi, S. Morid, M. Delavar, and R. Srinivasan, "Effect and side-effect assessment of different agricultural water saving measures in an integrated framework," Agricultural Water Management, vol. 223, Article ID 105685, 2019.

[23] M. Yu, T. Quan, Q. Peng, X. Yu, and L. Liu, "A model-based collaborate filtering algorithm based on stacked autoencoder," Neural Computing and Applications, vol. 4, 2021.

[24] P. Chen, T. Nie, S. Chen, Z. Zhang, Z. Qi, and W. Liu, "Recovery efficiency and loss of ${ }^{15} \mathrm{~N}$-labelled urea in a rice-soil system under water saving irrigation in the songnen plain of northeast China," Agricultural Water Management, vol. 222, 2019.

[25] X. Zhou, Y. Zhang, Z. Sheng et al., "Did water-saving irrigation protect water resources over the past 40 years? a global analysis based on water accounting framework," Agricultural Water Management, vol. 249, no. 7, Article ID 106793, 2021.

[26] Y. Zhao, R. L. Jia, and J. Wang, "Towards stopping land degradation in drylands: water-saving techniques for cultivating biocrusts in situ," Land Degradation \& Development, vol. 30, no. 18, pp. 2336-2346, 2019.

[27] H. Xuan, Q. Zheng, B. Li, and X. Wang, "A novel genetic simulated annealing algorithm for no-wait hybrid flowshop problem with unrelated parallel machines," ISIJ International, vol. 61, no. 1, pp. 258-268, 2021.

[28] W. Cen, J. Luo, J. Yu, and M. Shamin Rahman, "Slope stability analysis using genetic simulated annealing algorithm in conjunction with finite element method," KSCE Journal of Civil Engineering, vol. 24, no. 1, pp. 30-37, 2020.

[29] M. S. Yang, L. Ba, Y. Liu et al., "An improved genetic simulated annealing algorithm for stochastic two-sided assembly line balancing problem," International Journal of Simulation Modelling, vol. 18, no. 1, pp. 175-186, 2019.

[30] G. VenkataSubba Reddy, V. Ganesh, and C. Srinivasarao, "Cost reduction in clustering based unit commitment employing hybrid genetic-simulated annealing technique," Journal of Electrical Engineering \& Technology, vol. 14, no. 1, pp. 27-35, 2019.

[31] X. Yu, H. Su, Z. Fan, and Y. Dong, "Prediction of train wheel diameter based on Gaussian process regression optimized using a fast simulated annealing algorithm," PLoS One, vol. 14, Article ID e0226751, 2019.

[32] H. Amiry, R. Bendaoud, M. Benhmida, B. Zohal, and B. Elhadi, "New method for extracting physical parameters of PV generators combining an implemented genetic algorithm and the simulated annealing algorithm," Solar Energy, vol. 194, pp. 239-247, 2019.

[33] A. Fm, A. Dc, C. Klb, and A. Xz, "Multiple-class multidimensional knapsack optimisation problem and its solution approaches," Knowledge-Based Systems, vol. 166, pp. 1-17, 2019.

[34] M. Ramanauskas, D. Eok, J. Ilinskas, V. Starikoviius, A. Kaeniauskas, and R. Beleviius, "Global optimization of grillage-type foundations using a distributed genetic algorithm," Journal of Global Optimization, vol. 77, 2020.

[35] C. Jianli, C. Zhikui, W. Yuxin, and G. He, "Parallel genetic algorithm for $N$-Queens problem based on message passing interface-compute unified device architecture," Computational Intelligence, vol. 36, no. 4, pp. 1621-1637, 2020.

[36] K. Wang, X. Li, and L. Gao, "Modeling and optimization of multi-objective partial disassembly line balancing problem considering hazard and profit," Journal of Cleaner Production, vol. 211, pp. 115-133, 2019.

[37] Y. Yan, Y. Liang, H. Zhang et al., "A two-stage optimization method for unmanned aerial vehicle inspection of an oil and gas pipeline network," Petroleum Science, vol. 16, no. 2, pp. 458-468, 2019.

[38] Z. Fayyaz, N. Mohammadian, M. Reza Rahimi Tabar, R. Manwar, and K. Avanaki, "A comparative study of optimization algorithms for wavefront shaping," Journal of Innovative Optical Health Sciences, vol. 12, no. 4, Article ID 1942002, 2019.

[39] N. Y. Meitei, K. L. Baishnab, and G. Trivedi, "3D-IC partitioning method based on genetic algorithm," IET Circuits, Devices \& Systems, vol. 14, no. 7, pp. 1104-1109, 2020.

[40] K. Wang, X. Li, L. Gao, and P. Li, "Modeling and balancing for green disassembly line using associated parts precedence graph and multi-objective genetic simulated annealing," International Journal of Precision Engineering and Manufacturing-Green Technology, 2020.

[41] A. Ehsan, O. S. Bajgiran and E. Youssef, Using list-based simulated annealing and genetic algorithm for order batching and picker routing in put wall based picking systems," Applied Soft Computing, vol. 75, pp. 106-119, 2019.

[42] M. C. Morera, P. F. Monaghan, and M. D. Dukes, "Determinants of landscape irrigation water use in Florida-friendly yards," Environmental Management, vol. 65, no. 14, pp. 19-31, 2020.

[43] D. G. Hottinger, I. Nasr, J. K. Canner, D. Kattail, R. Koka, and D Schwengel, "Incidence, distribution, and cost of lawnmower injuries in the United States, 2006-2013," Public 
Health Reports, vol. 133, no. 6, pp. 570-577, Article ID 003335491878590, 2018.

[44] Z. Zhang, O. Kosareva, N. Zhang, L. Lin, and W. Liu, "Genetic algorithm for the location control of femtosecond laser filament," Scientific Reports, vol. 10, no. 1, Article ID 12878, 2020.

[45] S. Paek, S. Kim, and O. D. Weck, "Optimization of reconfigurable satellite constellations using simulated annealing and genetic algorithm," Sensors, vol. 19, no. 4, 2019. 